\title{
Derangement Polynomials and Excedances of Type $B$
}

\author{
William Y. C. Chen ${ }^{1}$, Robert L. Tang ${ }^{2}$ and Alina F. Y. Zhao ${ }^{3}$ \\ Center for Combinatorics, LPMC-TJKLC \\ Nankai University, Tianjin 300071, P. R. China \\ ${ }^{1}$ chen@nankai.edu.cn, ${ }^{2}$ tangling@cfc.nankai.edu.cn, ${ }^{3}$ zfeiyan@cf c.nankai.edu.cn
}

Submitted: Sep 4, 2008; Accepted: May 19, 2009; Published: Jun 10, 2009

Mathematics Subject Classifications: 05A15, 05A19

Dedicated to Anders Björner on the occasion of his sixtieth birthday

\begin{abstract}
Based on the notion of excedances of type $B$ introduced by Brenti, we give a type $B$ analogue of the derangement polynomials. The connection between the derangement polynomials and Eulerian polynomials naturally extends to the type $B$ case. Using this relation, we derive some basic properties of the derangement polynomials of type $B$, including the generating function formula, the Sturm sequence property, and the asymptotic normal distribution. We also show that the derangement polynomials are almost symmetric in the sense that the coefficients possess the spiral property.
\end{abstract}

\section{Introduction}

In this paper, we define a type $B$ analogue of the derangement polynomials by $q$-counting derangements with respect to the number of excedances of type $B$ introduced by Brenti [3]. We give some basic properties of these polynomials. It turns out that the connection between the derangement polynomials and the Eulerian polynomials naturally extends to the type $B$ case, where the type $B$ analogue of Eulerian polynomial has been given by Brenti [3], and has been further studied by Chow and Gessel in [7].

Let us now recall some definitions. Let $\mathfrak{S}_{n}$ be the set of permutations of $[n]=$ $\{1,2, \ldots, n\}$. For each $\sigma \in \mathfrak{S}_{n}$, the descent set and the excedance set of $\sigma=\sigma_{1} \sigma_{2} \cdots \sigma_{n}$ are defined as follows,

$$
\begin{aligned}
& \operatorname{Des}(\sigma)=\left\{i \in[n-1]: \sigma_{i}>\sigma_{i+1}\right\}, \\
& \operatorname{Exc}(\sigma)=\left\{i \in[n-1]: \sigma_{i}>i\right\} .
\end{aligned}
$$

The descent number and excedance number are defined by

$$
\operatorname{des}(\sigma)=|\operatorname{Des}(\sigma)|, \quad \operatorname{exc}(\sigma)=|\operatorname{Exc}(\sigma)| .
$$


The Eulerian polynomials $[10,14,16]$ are defined by

$$
A_{n}(q)=\sum_{\sigma \in \mathfrak{S}_{n}} q^{\operatorname{des}(\sigma)+1}=\sum_{\sigma \in \mathfrak{S}_{n}} q^{\operatorname{exc}(\sigma)+1}, \quad n \geq 1
$$

for $n=0$, we define $A_{0}(q)=1$. The Eulerian polynomials have the following generating function

$$
\sum_{n \geq 0} A_{n}(q) \frac{t^{n}}{n !}=\frac{(1-q) e^{q t}}{e^{q t}-q e^{t}}
$$

A permutation $\sigma=\sigma_{1} \sigma_{2} \cdots \sigma_{n}$ is a derangement if $\sigma_{i} \neq i$ for any $i \in[n]$. The set of derangements on $[n]$ is denoted by $D_{n}$. Brenti [1] defined the derangement polynomials of type $A$ by

$$
d_{n}(q)=\sum_{\sigma \in D_{n}} q^{\operatorname{exc}(\sigma)}, \quad n \geq 1
$$

and $d_{0}(q)=1$. It has been shown that $d_{n}(q)$ is symmetric and unimodal for $n \geq 1$. The following formula (1.2) is derived by Brenti [1].

Theorem 1.1 For $n \geq 0$,

$$
d_{n}(q)=\sum_{k=0}^{n}(-1)^{n-k}\left(\begin{array}{l}
n \\
k
\end{array}\right) \widetilde{A}_{k}(q)
$$

where

$$
\widetilde{A}_{n}(q)= \begin{cases}1, & \text { if } n=0 \\ \frac{1}{q} A_{n}(q), & \text { otherwise }\end{cases}
$$

The generating function of $d_{n}(q)$ has been obtained by Foata and Schützenberger [10], see, also, Brenti [1].

Theorem 1.2 We have

$$
\sum_{n \geq 0} d_{n}(q) \frac{t^{n}}{n !}=\frac{1}{1-\sum_{n \geq 2}\left(q+q^{2}+\cdots+q^{n-1}\right) t^{n} / n !} .
$$

A combinatorial proof of the above formula is given by Kim and Zeng [11] based on a decomposition of derangements. Brenti further proposed the conjecture that $d_{n}(q)$ has only real roots for $n \geq 1$, which has been proved independently by Zhang [17], and Canfield as mentioned in [2].

Theorem 1.3 The polynomials $\left\{d_{n}(q)\right\}_{n \geq 1}$ form a Sturm sequence. Precisely, for $n \geq 2$, $d_{n}(q)$ has $n-1$ distinct non-positive real roots, separated by the roots of $d_{n-1}(q)$.

The following recurrence relation is given by Zhang [17], which has been used to prove Theorem 1.3. 
Theorem 1.4 For $n \geq 2$, we have

$$
d_{n}(q)=(n-1) q d_{n-1}(q)+q(1-q) d_{n-1}^{\prime}(q)+(n-1) q d_{n-2}(q) .
$$

This paper is motivated by finding the right type $B$ analogue of the derangement polynomials. We find that the notion of excedances of type $B$ introduced by Brenti serves the purpose, although there are several possibilities to define type $B$ excedances, see $[3,6,15]$. It should be noted that the type $B$ derangement polynomials are not symmetric compared with type $A$ case. On the other hand, we will be able to show that they are almost symmetric in the sense that their coefficients have the spiral property.

This paper is organized as follows. In Section 2, we recall Brenti's definition of type $B$ excedances, and present the definition of derangement polynomials of type $B$, denoted by $d_{n}^{B}(q)$. Section 3 is concerned with the connection between the derangement polynomials of type $B$ and the Eulerian polynomials of type $B$. We derive a generating function formula for type $B$ derangement polynomials, and then extend the $U$-algorithm and $V$-algorithm given by Kim and Zeng [11] to derangements of type $B$. This gives a combinatorial interpretation of the generating function formula. In Section 4 , we prove that the polynomials $\left\{d_{n}^{B}(q)\right\}_{n \geq 1}$ form a Sturm sequence. Moreover, we show that the coefficients of $d_{n}^{B}(q)$ possess the spiral property. Section 5 is devoted to the limiting distribution of the coefficients of $d_{n}^{B}(q)$. By using Lyapunov's theorem we deduce that the distribution is normal.

\section{The Excedances of Type $B$}

In this section, we recall Brenti's definition of type $B$ excedances and give the definition of the derangement polynomials of type $B$. We adopt the notation and terminology on permutations of type $B$, or signed permutations, as given in [6]. Let $B_{n}$ be the hyperoctahedral group on $[n]$. We may regard the elements of $B_{n}$ as signed permutations of $[n]$, written as $\sigma=\sigma_{1} \sigma_{2} \cdots \sigma_{n}$, where some elements are associated with the minus sign. We may also express a negative element $-i$ in the form $\bar{i}$, and we will use $-\sigma$ to denote the signed permutation $\left(-\sigma_{1}\right)\left(-\sigma_{2}\right) \cdots\left(-\sigma_{n}\right)$.

The type $B$ descent set and the type $B$ ascent set of a signed permutation $\sigma$ are defined by

$$
\begin{aligned}
& \operatorname{Des}_{B}(\sigma)=\left\{i \in[0, n-1]: \sigma_{i}>\sigma_{i+1}\right\}, \\
& \operatorname{Asc}_{B}(\sigma)=\left\{i \in[0, n-1]: \sigma_{i}<\sigma_{i+1}\right\},
\end{aligned}
$$

where $\sigma_{0}=0$. The type $B$ descent and ascent numbers are given by

$$
\operatorname{des}_{B}(\sigma)=\left|\operatorname{Des}_{B}(\sigma)\right|, \quad \operatorname{asc}_{B}(\sigma)=\left|\operatorname{Asc}_{B}(\sigma)\right| .
$$

A derangement of type $B$ on $[n]$ is a signed permutation $\sigma=\sigma_{1} \sigma_{2} \cdots \sigma_{n}$ such that $\sigma_{i} \neq i$, for all $i \in[n]$. A fixed point of $\sigma$ is a position $i$ such that $\sigma_{i}=i$. The set of derangements in $B_{n}$ is denoted by $D_{n}^{B}$. 
Let us recall the definitions of excedances and weak excedances of type $B$ introduced by Brenti [3]. For further information on statistics on signed permutations, see [3, 7, 12].

Definition 2.1 Given $\sigma \in B_{n}$ and $i \in[n]$, we say that $i$ is a type $B$ excedance of $\sigma$ if $\sigma_{i}=-i$ or $\sigma_{\left|\sigma_{i}\right|}>\sigma_{i}$. We denote by $\operatorname{exc}_{B}(\sigma)$ the number of type $B$ excedances of $\sigma$. Similarly, we say that $i$ is a type $B$ weak excedance of $\sigma$ if $\sigma_{i}=i$ or $\sigma_{\left|\sigma_{i}\right|}>\sigma_{i}$, and we denote by $\operatorname{wexc}_{B}(\sigma)$ the number of type $B$ weak excedances of $\sigma$.

In view of the above definition of type $B$ excedances, we can define a type $B$ analogue of the derangement polynomials.

Definition 2.2 The type $B$ derangement polynomials $d_{n}^{B}(q)$ are defined by

$$
d_{n}^{B}(q)=\sum_{\sigma \in D_{n}^{B}} q^{\operatorname{exc}_{B}(\sigma)}=\sum_{k=0}^{n} d_{n, k} q^{k}, \quad n \geq 1,
$$

where $d_{n, k}$ is the number of derangements in $D_{n}^{B}$ with exactly $k$ excedances of type $B$. For $n=0$, we define $d_{0}^{B}(q)=1$.

Below are the polynomials $d_{n}^{B}(q)$ for $n \leq 10$ :

$$
\begin{aligned}
d_{1}^{B}(q)= & q, \\
d_{2}^{B}(q)= & 4 q+q^{2}, \\
d_{3}^{B}(q)= & 8 q+20 q^{2}+q^{3}, \\
d_{4}^{B}(q)= & 16 q+144 q^{2}+72 q^{3}+q^{4}, \\
d_{5}^{B}(q)= & 32 q+752 q^{2}+1312 q^{3}+232 q^{4}+q^{5}, \\
d_{6}^{B}(q)= & 64 q+3456 q^{2}+14576 q^{3}+9136 q^{4}+716 q^{5}+q^{6}, \\
d_{7}^{B}(q)= & 128 q+14912 q^{2}+127584 q^{3}+190864 q^{4}+55624 q^{5}+2172 q^{6}+q^{7}, \\
d_{8}^{B}(q)= & 256 q+62208 q^{2}+977920 q^{3}+2879232 q^{4}+2020192 q^{5} \\
& \quad+314208 q^{6}+6544 q^{7}+q^{8}, \\
d_{9}^{B}(q)= & 512 q+254720 q^{2}+6914816 q^{3}+35832320 q^{4}+49168832 q^{5} \\
& \quad+18801824 q^{6}+1697408 q^{7}+19664 q^{8}+q^{9}, \\
d_{10}^{B}(q)= & 1024 q+1032192 q^{2}+46429440 q^{3}+394153728 q^{4}+937670016 q^{5} \\
& \quad+704504832 q^{6}+161032224 q^{7}+8919456 q^{8}+59028 q^{9}+q^{10} .
\end{aligned}
$$




\section{$3 \quad$ The Generating Function}

In this section we obtain an expression of $d_{n}^{B}(q)$ in terms of $B_{n}(q)$, the Eulerian polynomials of type $B$. This formula is analogous to the formula of Brenti for the type $A$ case [1], and it enables us to derive the generating function of $d_{n}^{B}(q)$. Then we give a combinatorial interpretation of the generating function formula by extending the type $A$ argument of Kim and Zeng [11].

The Eulerian polynomials $B_{n}(q)$ are defined in terms of the number of descents of type $B$, see, Brenti [3],

$$
B_{n}(q)=\sum_{\sigma \in B_{n}} q^{\operatorname{des}_{B}(\sigma)}, \quad n \geq 1
$$

with $B_{0}(q)=1$.

Brenti [3] obtained the following formula for the generating function of the Eulerian polynomials of type $B$, see, also, Chow and Gessel [7],

$$
\sum_{n \geq 0} B_{n}(q) \frac{t^{n}}{n !}=\frac{(1-q) e^{t(1-q)}}{1-q e^{2 t(1-q)}} .
$$

The following theorem is obtained by Brenti [3] and it will be used to establish the formula for $d_{n}^{B}(q)$.

Theorem 3.1 There is a bijection $\varphi: B_{n} \rightarrow B_{n}$ such that

$$
\operatorname{asc}_{B}(\varphi(\sigma))=\operatorname{wexc}_{B}(\sigma)
$$

for any $\sigma \in B_{n}$.

The following relation indicates that the notion of excedances of type $B$ introduced by Brenti is a right choice for type $B$ derangement polynomials.

Theorem 3.2 We have

$$
d_{n}^{B}(q)=\sum_{k=0}^{n}(-1)^{n-k}\left(\begin{array}{l}
n \\
k
\end{array}\right) B_{k}(q)
$$

Proof. It is easy to see that

$$
\operatorname{des}_{B}(\sigma)=\operatorname{asc}_{B}(-\sigma)
$$

for all $\sigma \in B_{n}$. This implies that the number of descents and the number of ascents of type $B$ are equidistributed on $B_{n}$. On the other hand, Brenti [3] gave an involution $\alpha$ on $B_{n}$ such that $\operatorname{exc}_{B}(\sigma)=\operatorname{wexc}_{B}(\alpha(\sigma))$ for all $\sigma \in B_{n}$, where

$$
\alpha\left(\sigma_{i}\right)= \begin{cases}-\sigma_{i}, & \text { if }\left|\sigma_{i}\right|=i \\ \sigma_{i}, & \text { otherwise }\end{cases}
$$


It follows that the number of excedances of type $B$ and the number of weak excedances of type $B$ are equdistributed on $B_{n}$. By Theorem 3.1, we see that the number of excedances and the number of descents of type $B$ are equidistributed on $B_{n}$. Thus we deduce that

$$
B_{n}(q)=\sum_{\sigma \in B_{n}} q^{\operatorname{des}_{B}(\sigma)}=\sum_{\sigma \in B_{n}} q^{\operatorname{exc}_{B}(\sigma)} .
$$

We proceed to estalish the following relation

$$
\sum_{\pi \in B_{n}} q^{\operatorname{exc}_{B}(\pi)}=\sum_{k=0}^{n}\left(\begin{array}{l}
n \\
k
\end{array}\right) \sum_{\sigma \in D_{k}^{B}} q^{\operatorname{exc}_{B}(\sigma)}
$$

Like the cycle decomposition of an ordinary permutation, a signed permutation $\sigma$ can be expressed as a product of disjoint signed cycles, see, e.g., Brenti [3] and Chen [4]. For example, if $\sigma=\overline{6} 24 \overline{3} 15 \overline{7}$, then we can write $\sigma$ in the cycle form $\sigma=(1, \overline{6}, 5)(2)(4, \overline{3})(\overline{7})$. It is evident that a fixed point does not form an excedance of type $B$. Suppose that $\sigma$ contains $n-k$ fixed points. By removing the fixed points and reducing the remaining elements to $[k]$ by keeping the relative order, we get a derangement $\tau$ on $[k]$. It is easy to see that $\operatorname{exc}_{B}(\sigma)=\operatorname{exc}_{B}(\tau)$. For the $\sigma$ given above, we have $\tau=(1, \overline{5}, 4)(3, \overline{2})(\overline{6})$. Hence we obtain (3.5), that is,

$$
B_{n}(q)=\sum_{k=0}^{n}\left(\begin{array}{l}
n \\
k
\end{array}\right) d_{k}^{B}(q)
$$

By the binomial inversion, we arrive at (3.3). This completes the proof.

Using the generating function of $B_{n}(q)$, we derive the generating function of $d_{n}^{B}(q)$.

Theorem 3.3 We have

$$
\sum_{n \geq 0} d_{n}^{B}(q) \frac{t^{n}}{n !}=\frac{(1-q) e^{t q}}{e^{2 t q}-q e^{2 t}}=\frac{e^{t q}}{1-\sum_{n \geq 2} 2^{n}\left(q+q^{2}+\cdots+q^{n-1}\right) t^{n} / n !} .
$$

Proof. Using (3.2) and (3.6), we get

$$
e^{t} \sum_{n \geq 0} d_{n}^{B}(q) \frac{t^{n}}{n !}=\sum_{n \geq 0} B_{n}(q) \frac{t^{n}}{n !}=\frac{(1-q) e^{t(1-q)}}{1-q e^{2 t(1-q)}} .
$$

This gives (3.7).

Next, we give a combinatorial interpretation of the identity (3.7) based on an extension of the decomposition of derangements given by Kim and Zeng [11] in their combinatorial proof of (1.3).

Combinatorial Proof of Theorem 3.3. First, we give an outline of the proof of Kim and Zeng for derangements of type $A$. We adopt the convention that a cycle $\sigma=s_{1} s_{2} \cdots s_{k}$ of length $k$ is written in such a way that $s_{1}$ is the minimum element, $\sigma_{s_{i}}=s_{i+1}$ for $1 \leq i \leq k-1$, and $\sigma_{s_{k}}=s_{1}$. A cycle $\sigma$ (of length at least two) is called unimodal if 
there exists $i(2 \leq i \leq k)$ such that $s_{1}<\cdots<s_{i-1}<s_{i}>s_{i+1}>\cdots>s_{k}$. Moreover, a unimodal cycle $\sigma$ is called prime if it satisfies the additional condition $s_{i-1}<s_{k}$. It should be noted that a cycle with only one element is also considered as a unimodal and prime cycle. Let $\left(l_{1}, \ldots, l_{m}\right)$ be a composition of $n$, a sequence of prime cycles $\tau=\left(\tau_{1}, \tau_{2}, \ldots, \tau_{m}\right)$ is called a $P$-decomposition of type $\left(l_{1}, \ldots, l_{m}\right)$ if $\tau_{i}$ is of length $l_{i}$ and the underlying sets of $\tau_{1}, \tau_{2}, \ldots, \tau_{m}$ form a partition of $[n]$. Define the excedance of $\tau$ as the sum of the excedances of its prime cycles, that is,

$$
\operatorname{exc}(\tau)=\operatorname{exc}\left(\tau_{1}\right)+\cdots+\operatorname{exc}\left(\tau_{m}\right)
$$

and the weight of $\tau$ is defined by $q^{\operatorname{exc}(\tau)}$. Kim and Zeng found a bijection which maps the number of excedances of a derangement to the number of excedances of a $P$-decomposition of type $\left(l_{1}, \ldots, l_{m}\right), l_{i} \geq 2$. Then the generating function of $d_{n}(q)$ follows from the generating function of $P$-decomposition of type $\left(l_{1}, \ldots, l_{m}\right)$, as given by

$$
\left(\begin{array}{c}
l_{1}+\cdots+l_{m} \\
l_{1}, \ldots, l_{m}
\end{array}\right) \prod_{i=1}^{m}\left(q+\cdots+q^{l_{i}-1}\right) \frac{t^{l_{1}+\cdots+l_{m}}}{\left(l_{1}+\cdots+l_{m}\right) !} .
$$

Summing over $l_{1}, \ldots, l_{m} \geq 2$ and $m \geq 0$, we are led to the right hand side of the relation (1.3).

We now proceed to extend the above construction to type $B$ derangements. Observe that a signed permutation is a signed derangement if and only if the cycle decomposition does not have any one-cycle with a positive sign. More precisely, for any derangement $\pi$ of type $B$, we can decompose it into cycles

$$
\pi=\left(C_{1}, C_{2}, \ldots, C_{k}\right),
$$

where $C_{1}, C_{2}, \ldots, C_{k}$ are written in decreasing order of their minimum elements subject to the following order

$$
\bar{n}<\cdots<\overline{2}<\overline{1}<1<2<\cdots<n .
$$

Next we give two algorithms which help us to decompose each derangement of type $B$ into a $P$-decomposition with the same number of excedances of type $B$ to prove (3.7). The algorithm is described only for a cycle. Based on the cycle decomposition, one can apply the algorithm to transform a permutation into unimodal or prime cycles. Let us first describe the $U$-algorithm which transforms a permutation into unimodal cycles.

\section{The $U$-algorithm}

1. If $\sigma$ is unimodal, set $U(\sigma)=(\sigma)$.

2. Otherwise, let $i$ be the largest integer such that $s_{i-1}>s_{i}<s_{i+1}$ and $j$ be the unique integer greater than $i$ such that $s_{j}>s_{i}>s_{j+1}$. Set $U(\sigma)=\left(U\left(\sigma_{1}\right), \sigma_{2}\right)$, where $\sigma_{1}=s_{1} \cdots s_{i-1} s_{j+1} \cdots s_{k}$, and $\sigma_{2}=s_{i} s_{i+1} \cdots s_{j}$ is unimodal.

For example, let $\pi=3 \overline{5} 429 \overline{6} 87 \overline{1}$. Then we have $\operatorname{exc}_{B}(\pi)=5$, and $C_{1}=78$, $C_{2}=\overline{5} 9 \overline{1} 342$ and $C_{3}=\overline{6}$. Using the $U$-algorithm, we find

$$
U\left(C_{1}\right)=(78), \quad U\left(C_{2}\right)=(\overline{5} 9, \overline{1} 342), \quad U\left(C_{3}\right)=(\overline{6}),
$$


and

$$
U(\pi)=(78, \overline{5} 9, \overline{1} 342, \overline{6}) .
$$

Note that $\operatorname{exc}_{B}(U(\pi))=5$, which coincides with $\operatorname{exc}_{B}(\pi)=5$.

Next, we use the $V$-algorithm as given in [11], which transforms a sequence of unimodal cycles into a sequence of prime cycles by imposing the order relation (3.9).

\section{The $V$-algorithm}

1. If $\sigma$ is prime, then set $V(\sigma)=(\sigma)$.

2. Otherwise, let $j$ be the smallest integer such that $s_{j}>s_{i}>s_{j+1}>s_{i-1}$ for some integer $i$ greater than 1 . Then set $V(\sigma)=\left(V\left(\sigma_{1}\right), \sigma_{2}\right)$, where $\sigma_{1}=s_{1} \cdots s_{i-1} s_{j+1} \cdots s_{k}$, and $\sigma_{2}=s_{i} s_{i+1} \cdots s_{j}$ is prime.

Applying $V$-algorithm to each cycle of $U(\pi)$ in the above example, we obtain that

$$
V(U(\pi))=(78, \overline{5} 9, \overline{1} 2,34, \overline{6}) .
$$

One can check that $\operatorname{exc}_{B}(V(U(\pi)))=5$.

Combining the $U$-algorithm and the $V$-algorithm, we can transform a derangement in $B_{n}$ to a $P$-decomposition of $[n]$. Assume that $\left|s_{t-2}\right|$ is an excedance of type $B$ of the signed cycle $\sigma=s_{1} s_{2} \cdots s_{k}$, namely, $\sigma_{\left|\sigma_{\left|s_{t-2}\right|}\right|}>\sigma_{\left|s_{t-2}\right|}$. In light of the cycle notation of $\sigma$, we have $\sigma_{\left|s_{t-2}\right|}=s_{t-1}, \sigma_{\left|\sigma_{\left|s_{t-2}\right|}\right|}=s_{t}$ and $s_{t}>s_{t-1}$. Thus the number of excedances of type $B$ in a cycle $\sigma$ of length larger than two equals the number of indices $i$ such that $s_{i}>s_{i+1}$. As long as the order is given, it is the same as counting the number of excedances of an ordinary cycle. This implies that as the type $A$ case, the number of excedances of type $B$ in $\pi$ equals to the total number of excedances of type $B$ in all prime (resp. unimodal) cycles. In the type $B$ case, we define the weight of each prime cycle $\tau$ by $q^{\operatorname{exc}_{B}(\tau)}$. Notice that in the cycle decomposition of a type $B$ derangement, we allow cycles of length one with negative elements. Thus the corresponding $P$-decompositions have type $\left(1^{k}, l_{1}, \ldots, l_{m}\right), k \geq 0, l_{i} \geq 2$. For a cycle containing only one negative element, the weight is $q$. For a cycle of length $l \geq 2$, we have $2^{l}$ choices for the $l$ elements in the prime

cycle, so the weight of such a prime cycle on a given $l$-set is $2^{l}\left(q+q^{2}+\cdots+q^{l-1}\right)$. Hence the generating function of $d_{n}^{B}(q)$ follows from the generating function of $P$-decompositions of type $\left(1^{k}, l_{1}, \ldots, l_{m}\right), k \geq 0, l_{i} \geq 2$, as given by

$$
q^{k} t^{k}\left(\begin{array}{c}
l_{1}+\cdots+l_{m} \\
l_{1}, \ldots, l_{m}
\end{array}\right) \prod_{i=1}^{m} 2^{l_{i}}\left(q+\cdots+q^{l_{i}-1}\right) \frac{t^{l_{1}+\cdots+l_{m}}}{\left(l_{1}+\cdots+l_{m}\right) !} .
$$

Summing over $l_{1}, \ldots, l_{m} \geq 2$ and $k \geq 0, m \geq 0$, we obtain the right hand side of (3.7).

\section{A Recurrence Relation}

In this section, we use the recurrence relation for Eulerian polynomials of type $B$ to derive a recurrence relation for the derangement polynomials $d_{n}^{B}(q)$. Applying a theorem 
of Zhang [18], we deduce that the polynomials $\left\{d_{n}^{B}(q)\right\}_{n \geq 1}$ form a Sturm sequence, that is, $d_{n}^{B}(q)$ has only real roots which are separated by the roots of $d_{n-1}^{B}(q)$. Moreover, from the initial values, one sees that $d_{n}^{B}(q)$ has only non-positive real roots for any $n \geq 1$. Consequently, $d_{n}^{B}(q)$ is log-concave. Although the polynomials $d_{n}^{B}(q)$ are not symmetric, we show that they are almost symmetric in the sense that the coefficients have the spiral property.

The following recurrence formula $(4.1)$ for $B_{n}(q)$ is a special case of Theorem 3.4 in Brenti [3], see, also, Chow and Gessel [7]. This relation leads to a recurrence for $d_{n}^{B}(q)$.

Theorem 4.1 For $n \geq 1$, we have

$$
B_{n}(q)=((2 n-1) q+1) B_{n-1}(q)+2 q(1-q) B_{n-1}^{\prime}(q) .
$$

Theorem 4.2 For $n \geq 2$, we have

$$
d_{n}^{B}(q)=(2 n-1) q d_{n-1}^{B}(q)+2 q(1-q) d_{n-1}^{B^{\prime}}(q)+2(n-1) q d_{n-2}^{B}(q) .
$$

Proof. By (3.3) and (4.1), we obtain

$$
\begin{aligned}
d_{n}^{B}(q)= & \sum_{k=0}^{n}(-1)^{n-k}\left(\begin{array}{l}
n \\
k
\end{array}\right) B_{k}(q) \\
= & \sum_{k=0}^{n}(-1)^{n-k}\left(\left(\begin{array}{c}
n-1 \\
k-1
\end{array}\right)+\left(\begin{array}{c}
n-1 \\
k
\end{array}\right)\right) B_{k}(q) \\
= & -d_{n-1}^{B}(q)+\sum_{k=1}^{n}(-1)^{n-k}\left(\begin{array}{c}
n-1 \\
k-1
\end{array}\right)\left(((2 k-1) q+1) B_{k-1}(q)+2 q(1-q) B_{k-1}^{\prime}(q)\right) \\
= & -q d_{n-1}^{B}(q)+2 q \sum_{k=1}^{n}(-1)^{n-k}\left(\left(\begin{array}{c}
n \\
k
\end{array}\right)-\left(\begin{array}{c}
n-1 \\
k
\end{array}\right)\right) k B_{k-1}(q)+2 q(1-q) d_{n-1}^{B^{\prime}}(q) \\
= & -d_{n-1}^{B}(q)+2 n q \sum_{k=1}^{n}(-1)^{n-k}\left(\begin{array}{c}
n-1 \\
k-1
\end{array}\right) B_{k-1}(q) \\
& \quad+2 q(n-1) \sum_{k=1}^{n}(-1)^{n-k-1}\left(\begin{array}{c}
n-2 \\
k-1
\end{array}\right) B_{k-1}(q)+2 q(1-q) d_{n-1}^{B^{\prime}}(q) \\
= & (2 n-1) q d_{n-1}^{B}(q)+2(n-1) q d_{n-2}^{B}(q)+2 q(1-q) d_{n-1}^{B^{\prime}}(q),
\end{aligned}
$$

as desired.

Equating coefficients on both sides of (4.2), we are led to the following recurrence relation for the numbers $d_{n, k}$.

Corollary 4.3 For $n \geq 2$ and $k \geq 1$, we have

$$
d_{n, k}=2 k d_{n-1, k}+(2 n-2 k+1) d_{n-1, k-1}+2(n-1) d_{n-2, k-1} .
$$

From the above relation (4.3), it follows that $d_{n, 1}=2^{n}$ for $n>1$. The recurrence relation (4.2) enables us to show that the polynomials $\left\{d_{n}^{B}(q)\right\}_{n \geq 1}$ form a Sturm sequence. The proof turns out to be an application of the following theorem of Zhang [18]. 
Theorem 4.4 Let $f_{n}(q)$ be a polynomial of degree $n$ with nonnegative real coefficients satisfying the following conditions:

(1) For $n \geq 2, f_{n}(q)=a_{n} q f_{n-1}(q)+b_{n} q\left(1+c_{n} q\right) f_{n-1}^{\prime}(q)+d_{n} q f_{n-2}(q)$, where $a_{n}>$ $0, b_{n}>0, c_{n} \leq 0, d_{n} \geq 0$

(2) For $n \geq 1$, zero is a simple root of $f_{n}(q)$;

(3) $f_{0}(q)=e, f_{1}(q)=e_{1} q$ and $f_{2}(q)$ has two real roots, where $e \geq 0$ and $e_{1} \geq 0$.

Then for $n \geq 2$, the polynomial $f_{n}(q)$ has $n$ distinct real roots, separated by the roots of $f_{n-1}(q)$.

It can be easily verified that the recurrence relation (4.2) satisfies the conditions in the above theorem. Thus we reach the following assertion.

Theorem 4.5 The polynomials $\left\{d_{n}^{B}(q)\right\}_{n \geq 1}$ form a Sturm sequence, that is, for $n \geq 2$, $d_{n}^{B}(q)$ has $n$ distinct non-positive real roots, separated by the roots of $d_{n-1}^{B}(q)$.

As a consequence of the above theorem, we see that the coefficients of $d_{n}^{B}(q)$ are logconcave for $n \geq 1$. We will show that the coefficients of $d_{n}^{B}(q)$ satisfy the spiral property. This property was first observed by Zhang [19] in his proof of a conjecture of Chen and Rota [5].

Theorem 4.6 The polynomials $d_{n}^{B}(q)$ possess the spiral property. Precisely, for $n \geq 2$, if $n$ is even,

$$
d_{n, n}<d_{n, 1}<d_{n, n-1}<d_{n, 2}<d_{n, n-2}<\cdots<d_{n, \frac{n}{2}+2}<d_{n, \frac{n}{2}-1}<d_{n, \frac{n}{2}+1}<d_{n, \frac{n}{2}},
$$

and if $n$ is odd,

$$
d_{n, n}<d_{n, 1}<d_{n, n-1}<d_{n, 2}<d_{n, n-2}<\cdots<d_{n, \frac{n+3}{2}}<d_{n, \frac{n-1}{2}}<d_{n, \frac{n+1}{2}} .
$$

Proof. Let

$$
f(n)= \begin{cases}\frac{n}{2}-1, & \text { if } n \text { is even, } \\ \frac{n-1}{2}, & \text { if } n \text { is odd. }\end{cases}
$$

In this notation, the spiral property can be described by the following inequalities

$$
d_{n, n+1-k}<d_{n, k}<d_{n, n-k}
$$

for any $1 \leq k \leq f(n)$, and the inequality

$$
d_{n, \frac{n}{2}+1}<d_{n, \frac{n}{2}}
$$

when $n$ is even. 
We proceed to prove the relations (4.4) and (4.5) by induction on $n$. It is easily seen that (4.4) and (4.5) hold for $n=2$ and $n=3$. We now assume that they hold for all integers up to $n$. We claim that

$$
d_{n+1, n+2-k}<d_{n+1, k}<d_{n+1, n+1-k}
$$

for any $1 \leq k \leq f(n+1)$. We will also show that when $n+1$ is even,

$$
d_{n+1, \frac{n+3}{2}}<d_{n+1, \frac{n+1}{2}} \text {. }
$$

For $k=1$, we have $d_{n+1, n+1}-d_{n+1,1}=1-2^{n+1}<0$. For $2 \leq k \leq f(n+1)$, by the recurrence relation (4.3) for $d_{n, k}$, we have

$$
\begin{aligned}
d_{n+1, n+2-k} & =2(n+2-k) d_{n, n+2-k}+(2 k-1) d_{n, n+1-k}+2 n d_{n-1, n+1-k}, \\
d_{n+1, k} & =2 k d_{n, k}+(2 n-2 k+3) d_{n, k-1}+2 n d_{n-1, k-1}, \\
d_{n+1, n+1-k} & =2(n+1-k) d_{n, n+1-k}+(2 k+1) d_{n, n-k}+2 n d_{n-1, n-k} .
\end{aligned}
$$

It follows from (4.8) and (4.9) that

$$
\begin{aligned}
d_{n+1, n+2-k}-d_{n+1, k}= & (2 n-2 k+3)\left(d_{n, n+2-k}-d_{n, k-1}\right)+2 k\left(d_{n, n+1-k}-d_{n, k}\right) \\
& +2 n\left(d_{n-1, n+1-k}-d_{n-1, k-1}\right)+\left(d_{n, n+2-k}-d_{n, n+1-k}\right) .
\end{aligned}
$$

By the inductive hypothesis, we see that the difference in every parenthesis in the above expression is negative. This implies that for $2 \leq k \leq f(n+1)$

$$
d_{n+1, n+2-k}-d_{n+1, k}<0 .
$$

Similarly, for $2 \leq k \leq f(n+1)$, in view of (4.9) and (4.10) we find

$$
\begin{aligned}
d_{n+1, k}-d_{n+1, n+1-k}= & (2 k+1)\left(d_{n, k}-d_{n, n-k}\right)+2 n\left(d_{n-1, k-1}-d_{n-1, n-k}\right) \\
& +(2 n+3-2 k)\left(d_{n, k-1}-d_{n, n+1-k}\right)+\left(d_{n, n+1-k}-d_{n, k}\right) .
\end{aligned}
$$

Again, by the inductive hypothesis, we deduce that for $2 \leq k \leq f(n+1)$,

$$
d_{n+1, k}-d_{n+1, n+1-k}<0 .
$$

Combining (4.11) and (4.12) gives (4.6) for $1 \leq k \leq f(n+1)$.

It remains to verify (4.7) when $n+1$ is even. By the recurrence relation (4.3), we have

$$
\begin{aligned}
& d_{n+1, \frac{n+3}{2}}=(n+3) d_{n, \frac{n+3}{2}}+n d_{n, \frac{n+1}{2}}+2 n d_{n-1, \frac{n+1}{2}} \\
& d_{n+1, \frac{n+1}{2}}=(n+1) d_{n, \frac{n+1}{2}}+(n+2) d_{n, \frac{n-1}{2}}+2 n d_{n-1, \frac{n-1}{2}} .
\end{aligned}
$$

This yields

$$
\begin{aligned}
d_{n+1, \frac{n+3}{2}}-d_{n+1, \frac{n+1}{2}}=( & +2)\left(d_{n, \frac{n+3}{2}}-d_{n, \frac{n-1}{2}}\right)+\left(d_{n, \frac{n+3}{2}}-d_{n, \frac{n+1}{2}}\right) \\
+ & 2 n\left(d_{n-1, \frac{n+1}{2}}-d_{n-1, \frac{n-1}{2}}\right) .
\end{aligned}
$$

Again, by the inductive hypothesis, we obtain (4.7). This completes the proof. 


\section{The Limiting Distribution}

In this section, we show that the limiting distribution of the coefficients of $d_{n}^{B}(q)$ is normal. The type $A$ case has been studied by Clark [9]. It has been shown that the limiting distribution of the coefficients of $d_{n}(q)$ is normal. Let $\xi_{n}$ be the number of type $B$ excedances in a random type $B$ derangement on $[n]$. We first compute the expectation and the variance of $\xi_{n}$. Then we use Lyapunov's theorem to show that $\xi_{n}$ is asymptotically normal.

Theorem 5.1 We have

$$
\begin{aligned}
\mathrm{E} \xi_{n} & =\frac{n}{2}+\frac{1}{4}+o(1), \\
\operatorname{Var} \xi_{n} & =\frac{n}{12}-\frac{1}{16}+o(1) .
\end{aligned}
$$

Proof. By the recurrence relation (4.1) for $B_{n}(x)$, we have for $n \geq 1$,

$$
B_{n}^{\prime}(x)=(2 n-1) B_{n-1}(x)+(2 n x-5 x+3) B_{n-1}^{\prime}(x)+2 x(1-x) B_{n-1}^{\prime \prime}(x) .
$$

Since $B_{n}(1)=2^{n} n$ ! for $n \geq 0$, setting $x=1$ in (5.3) gives the following recurrence relation for $B_{n}^{\prime}(1)$ :

$$
B_{n}^{\prime}(1)=(2 n-1)(n-1) ! 2^{n-1}+(2 n-2) B_{n-1}^{\prime}(1) .
$$

It can be verified that for $n \geq 1$,

$$
B_{n}^{\prime}(1)=\frac{n 2^{n} n !}{2}
$$

Moreover, by (5.3) we get

$$
B_{n}^{\prime \prime}(x)=(4 n-6) B_{n-1}^{\prime}(x)+(2 n x-9 x+5) B_{n-1}^{\prime \prime}(x)+2 x(1-x) B_{n-1}^{\prime \prime \prime}(x) .
$$

Setting $x=1$ in (5.5) and using (5.4), we obtain

$$
B_{n}^{\prime \prime}(1)=(2 n-3)(n-1) 2^{n-1}(n-1) !+(2 n-4) B_{n-1}^{\prime \prime}(1) .
$$

One can check that the solution of the above recurrence relation is given by

$$
B_{n}^{\prime \prime}(1)=\frac{\left(3 n^{2}-5 n+1\right) 2^{n} n !}{12}, \quad n \geq 2 .
$$

Since $B_{n}(1)=2^{n} n$ !, in view of the formula (3.3), we see that

$$
d_{n}^{B}(1)=\sum_{k=0}^{n}(-1)^{n-k}\left(\begin{array}{l}
n \\
k
\end{array}\right) B_{k}(1) .
$$

Let

$$
s_{n}=\sum_{k=0}^{n}(-1)^{k} \frac{1}{2^{k} \cdot k !} .
$$


So $d_{n}^{B}(1)$ can be written as $2^{n} n ! s_{n}$.

Applying the formula (3.3) again and using the evaluation (5.4) for $B_{n}^{\prime}(1)$, we find that for $n \geq 1$,

$$
\begin{aligned}
d_{n}^{B^{\prime}}(1) & =\sum_{m=1}^{n}(-1)^{n-m}\left(\begin{array}{c}
n \\
m
\end{array}\right) \cdot 2^{m-1} m \cdot m ! \\
& =2^{n} n ! \sum_{m=0}^{n-1}(-1)^{m} \frac{n-m}{m ! 2^{m+1}} \\
& =2^{n} n !\left(\frac{n}{2} \sum_{m=0}^{n-1}(-1)^{m} \frac{1}{m ! 2^{m}}+\frac{1}{4} \sum_{m=0}^{n-1}(-1)^{m-1} \frac{1}{2^{m-1}(m-1) !}\right) \\
& =\frac{2^{n} n !}{2}\left(n s_{n-1}+\frac{1}{2} s_{n-2}\right) .
\end{aligned}
$$

Differentiating (3.3) twice and invoking (5.7), we deduce that for $n \geq 2$

$$
\begin{aligned}
d_{n}^{B^{\prime \prime}}(1) & =\sum_{m=2}^{n}(-1)^{n-m}\left(\begin{array}{c}
n \\
m
\end{array}\right) 2^{m} m ! \frac{3 m^{2}-5 m+1}{12} \\
& =\frac{2^{n} n !}{12} \sum_{m=2}^{n}(-1)^{n-m} \frac{3 m^{2}-5 m+1}{(n-m) ! 2^{n-m}} \\
& =\frac{2^{n} n !}{12} \sum_{m=0}^{n-2}(-1)^{m} \frac{3(n-m)^{2}-5(n-m)+1}{m ! 2^{m}} \\
& =\frac{2^{n} n !}{12}\left(\sum_{m=0}^{n-2}(-1)^{m} \frac{3 n^{2}-5^{n}+1}{m ! 2^{m}}+\sum_{m=0}^{n-2}(-1)^{m} \frac{-6 n+5}{(m-1) ! 2^{m}}+3 \sum_{m=0}^{n-2}(-1)^{m} \frac{m^{2}}{m ! 2^{m}}\right) \\
& =\frac{2^{n} n !}{12}\left(\left(3 n^{2}-5 n+1\right) s_{n-2}+\frac{1}{2}(6 n-5) s_{n-3}+\frac{3}{4} s_{n-4}-\frac{3}{2} s_{n-3}\right) \\
& =\frac{2^{n} n !}{12}\left(\left(3 n^{2}-5 n+1\right) s_{n-2}+(3 n-4) s_{n-3}+\frac{3}{4} s_{n-4}\right) .
\end{aligned}
$$

It is easy to see that $s_{n-r} / s_{n}=1+o(1)$ for $r=1,2,3,4$. From (5.8), (5.9) and (5.10), we conclude that

$$
\begin{aligned}
& \mathrm{E} \xi_{n}=\frac{d_{n}^{B^{\prime}}(1)}{d_{n}^{B}(1)}=\frac{n}{2}+\frac{1}{4}+o(1), \\
& \operatorname{Var} \xi_{n}=\frac{d_{n}^{B^{\prime \prime}}(1)}{d_{n}^{B}(1)}+\mathrm{E} \xi_{n}-\left(\mathrm{E} \xi_{n}\right)^{2}=\frac{n}{12}-\frac{1}{16}+o(1),
\end{aligned}
$$

as desired.

Given the formulas for the expectation and variance of $\xi_{n}$, we will use Lyapunov's theorem [13, Section 1.2] to show that the limiting distribution of $\xi_{n}$ is normal. Recall 
that a triangular array of independent random variables $\xi_{n, k}, k=1,2, \ldots, n, n=1,2, \ldots$, is called a Poisson sequence if

$$
P\left\{\xi_{n, k}=1\right\}=p_{k}, \quad P\left\{\xi_{n, k}=0\right\}=q_{k},
$$

where $p_{k}=p_{k}(n) \in[0,1], q_{k}=q_{k}(n)$ and $p_{k}+q_{k}=1$. Lyapunov's theorem can be used to derive asymptotically normal distributions.

Theorem 5.2 (Lyapunov) Let

$$
V_{n}^{2}=\sum_{k=1}^{n} p_{k} q_{k}, \quad \eta_{n}=V_{n}^{-1} \sum_{k=1}^{n}\left(\xi_{n, k}-p_{k}\right) .
$$

If $V_{n} \rightarrow \infty$ as $n \rightarrow \infty$, then the sequence $\left\{\eta_{n}\right\}$ is asymptotically standard normal.

The above theorem enables us to derive the asymptotic distribution of the random variable $\eta_{n}$.

Theorem 5.3 The distribution of the random variable

$$
\eta_{n}=\frac{\xi_{n}-\mathrm{E} \xi_{n}}{\sqrt{\operatorname{Var} \xi_{n}}}
$$

converges to the standard normal distribution as $n \rightarrow \infty$.

Proof. Since the polynomials $d_{n}^{B}(q)$ have distinct, real and non-positive roots, we may express $d_{n}^{B}(q)$ as

$$
d_{n}^{B}(q)=q\left(q+\alpha_{1}\right)\left(q+\alpha_{2}\right) \cdots\left(q+\alpha_{n-1}\right),
$$

where $\alpha_{i}>0$ for all $1 \leq i \leq n-1$. Obviously, $P\left(\xi_{n}=k\right)=d_{n, k} / d_{n}^{B}(1)$. The probability generating function

$$
P_{n}(x)=\sum_{k=1}^{n} P\left(\xi_{n}=k\right) x^{k}
$$

can be easily written as

$$
\begin{aligned}
P_{n}(x) & =\frac{x\left(x+\alpha_{1}\right)\left(x+\alpha_{2}\right) \cdots\left(x+\alpha_{n-1}\right)}{\left(1+\alpha_{1}\right)\left(1+\alpha_{2}\right) \cdots\left(1+\alpha_{n-1}\right)} \\
& =x\left(\frac{x}{1+\alpha_{1}}+\frac{\alpha_{1}}{1+\alpha_{1}}\right) \cdots\left(\frac{x}{\alpha_{n-1}}+\frac{\alpha_{n-1}}{1+\alpha_{n-1}}\right) .
\end{aligned}
$$

Consider the independent random variables $\xi_{n, 1}, \xi_{n, 2}, \ldots, \xi_{n, n}$, taking the values 0 and 1 , such that

$$
P\left(\xi_{n, k}=1\right)=p_{k}=\frac{1}{1+\alpha_{k}} \in[0,1], \quad k=1,2, \ldots, n
$$


with the convention that $\alpha_{n}=0$. It is evident that the variance of $\xi_{n, k}$ equals $p_{k}\left(1-p_{k}\right)$. Hence the random variable $\xi_{n}$, namely, the number of type $B$ excedances in a random type $B$ derangement on $[n]$, can be represented as a sum of independent random variables

$$
\xi_{n}=\xi_{n, 1}+\xi_{n, 2}+\cdots+\xi_{n, n-1}+\xi_{n, n} .
$$

Since the variables $\xi_{n, 1}, \xi_{n, 2}, \ldots, \xi_{n, n}$ are independent, we obtain that

$$
\operatorname{Var}\left(\xi_{n}\right)=\sum_{k=1}^{n} \operatorname{Var}\left(\xi_{n, k}\right)=\sum_{k=1}^{n} p_{k}\left(1-p_{k}\right) .
$$

On the other hand, it follows from (5.12) that $\operatorname{Var}\left(\xi_{n}\right) \rightarrow \infty$ as $n \rightarrow \infty$. By Theorem 5.2, we reach the conclusion that $\eta_{n}$ is asymptotically standard normal.

Note added in proof. Chow [8] defined the derangement polynomials of type $B$ based on the number of weak excedances. As pointed out by Chow [8], the number of excedances and $n$ minus the number of weak excedances of type $B$ are equidistributed over derangements of type $B$, the derangement polynomials of type $B$ defined in this paper is essentially the same as the polynomials defined by Chow. By different methods, Chow has independently obtained the generating function, recurrence relation, real-rootedness. Theorem 4.2, Theorem 3.3 and Theorem 4.5 in this paper are equivalent to Proposition 3.1, Theorem 3.2 and Theorem 3.3 in Chow [8], respectively.

Acknowledgments. The authors would like to thank the referees for helpful comments leading to an improvement of an earlier version. This work was supported by the 973 Project, the PCSIRT Project of the Ministry of Education, the Ministry of Science and Technology, and the National Science Foundation of China.

\section{References}

[1] F. Brenti, Unimodal polynomials arising from symmetric functions, Proc. Amer. Math. Soc. 108 (1990), 1133-1141.

[2] F. Brenti, Permutation enumeration, symmetric functions and unimodality, Pacific J. Math. 157 (1993), 1-28.

[3] F. Brenti, $q$-Eulerian polynomials arising from Coxeter groups, European J. Combin. 15 (1994), 417-441.

[4] W.Y.C. Chen, Induced cycle structures of the hyperoctahedral group, SIAM J. Discrete Math. 6 (1993), 353-362.

[5] W.Y.C. Chen and G.-C. Rota, $q$-Analogs of the inclusion-exclusion principle and permutations with restricted position, Discrete Math. 104 (1992), 7-22.

[6] C.-O. Chow, On derangement polynomials of type B, Sém. Lothar. Combin. 55 (2006), Artical B55b. 
[7] C.-O. Chow and I.M. Gessel, On the descent numbers and major indices for the hyperoctahedral group, Adv. Appl. Math. 38 (2007), 275-301.

[8] C.-O. Chow, On derangement polynomials of type B. II, J. Combin. Theory Ser. A 116 (2009), 816-830.

[9] L. Clark, Central and local limit theorems for excedances by conjugacy class and by derangement, Integers 2 (2002), Paper A3.

[10] D. Foata and M.P. Schützenberger, Théorie géométrique des polynômes eulériens, Lecture Notes in Math. Vol. 138, Springer-Verlag, Berlin, 1970.

[11] D. Kim and J. Zeng, A new decomposition of derangements, J. Combin. Theory Ser. A 96 (2001), 192-198.

[12] V. Reiner, Signed permutation statistics, European J. Combin. 14 (1993), 553-567.

[13] V.N. Sachkov, Probabilistic Methods in Combinatorial Analysis, Cambridge University Press, New York, 1997.

[14] R.P. Stanley, Enumerative Combinatorics, Vol. 1, Cambridge University Press, Cambridge, 1997.

[15] E. Steingrímsson, Indexed permutations and poset permutations, Ph.D. Thesis, MIT, 1991.

[16] J. Shareshian and M.L. Wachs, $q$-Eulerian polynomials: Excedance number and major index, Electron. Res. Announc. Amer. Math. Soc. 13 (2007), 33-45.

[17] X.D. Zhang, On q-derangement polynomials, Combinatorics and Graph Theory 95, Vol. 1 (Hefei), World Sci. Publishing, River Edge, NJ, 1995, pp. 462-465,

[18] X.D. Zhang, On a kind of sequence of polynomials, in: Computing and Combinatorics, Xi'an, 1995, in: Lecture Notes in Comput. Sci., vol. 959, Springer, Berlin, 1995, 379-383.

[19] X.D. Zhang, Note on the spiral property of the $q$-derangement numbers, Discrete Math. 159 (1996), 295-298. 